\title{
Canaux de jonction intercellulaires, différenciation, cancer et horloges biologiques
}

Il existe, dans les tissus organisés aussi bien que dans des cultures de cellules confluentes, des canaux intercellulaires faisant communiquer directement les cytosols de deux cellules adjacentes. Leur diamètre moyen .est, chez les mammifères, de 16 à $20 \mathrm{~A}$ et ils sont constitués de sous-unités protéiques de $32 \mathrm{kDa}$ provenant pour moitié de chacune des cellules en communication. Un ADN complémentaire du messager d'une sous-unité de tels canaux a été isolé à partir de foie et la séquence de la protéine a été déduite de la séquence nucléotidique. Les canaux intercellulaires jonctionnels (gap junctions en anglais) sont normalement dans un état ouvert stable qui les différencie nettement des canaux ioniques dont l'oscillation continuelle entre les états ouverts et fermés est une importante caractéristique [1]. Leurs rôles sont multiples. Dans le cerveau, ils constituent des "synapses électriques "impliquées dans la conduction électrotonique de l'influx nerveux entre deux cellules via le passage d'ions. Un phénomène semblable est bien connu dans le cœur. Les canaux jonctionnels peuvent conduire à une amplification et à une synchronisation au niveau d'un ensemble cellulaire complexe du signal transmis via la libération de neuromédiateurs dans une synapse chimique; ils peuvent, par conséquent, constituer un chaînon important d'activités électriques oscillatoires au niveau de cellules nerveuses. Une telle fonction expliquerait bien qu'ils interviennent dans la régulation des " horloges moléculaires " contrôlant les rythmes biologiques et comportementaux, comme le suggèrent les derniers résultats d'une équipe de $\mathrm{New}$ York sur la mutation "per" de la drosophile [2]. Nous avons plusieurs fois parlé dans $\mathrm{m} / \mathrm{s}$ de cette mutation qui perturbe un ensemble complexe de rythmes biologiques de la larve aussi bien que de l'insecte adulte $\left(\mathrm{m} / \mathrm{s} n^{\circ} 4, \mathrm{vol}\right.$. 2, p. 223 et $n^{\circ} 6$, vol. 2, p. 340). Les mutants per, arythmiques, ont une diminution considérable du nombre des canaux jonctionnels au niveau des glandes salivaires, alors que les mutants per', à rythmes augmentés, présentent une importante augmentation du nombre de ces canaux. Le gène per, qui code pour une protéine de type "protéoglycane", pourrait donc contrôler, en fait, l'établissement de canaux intercellulaires intervenant dans la synchronisation d'un oscillateur biologique.

Dans un tout autre modèle, celui d'hépatocytes de rats adultes en culture, il vient d'ailleurs d'être montré que différents types de protéoglycanes des matrices extracellulaires stimulaient considérablement l'établissement de canaux jonctionnels... et amélioraient la transcription par les cellules en culture de gènes codant pour des marqueurs spécifiques de la différenciation hépatique [3]. Une relation entre l'établissement des communications intercellulaires d'une part, le développement et la différenciation d'autre part, existe pour bien d'autres tissus que le foie. Il est probable que des morphogènes de petit poids moléculaire peuvent diffuser par de tels canaux, qui joueraient ainsi un rôle essentiel dans l'embryogenèse. Il est d'ailleurs possible de perturber la morphogenèse de cellules filles dérivées d'une cellule d'un embryon précoce de Xénope (stade 8 cellules) dans laquelle des anticorps dirigés contre la protéine des canaux jonctionnels ont été injectés (cité dans [1]).

Des seconds messagers de l'action hormonale peuvent, peut-être, aussi diffuser par les jonctions intercellulaires et conférer à ces jonctions un rôle dans la régula- tion de la fonction de certains tissus. Une telle intervention des canaux intercellulaires sur la fonction de cellules glandulaires a été récemment suggérée pour le pancréas exocrine où une corrélation existe entre la sécrétion d'amylase et le blocage des communications intercellulaires [4]. Une conséquence importante de l'existence des canaux intercellulaires est de rendre, d'une certaine manière, les cellules adjacentes solidaires les unes des autres... pour le meilleur et pour le pire. Le pire, c'est la possibilité que la souffrance d'une cellule et les altérations ioniques et métaboliques (acidose, par exemple) qui la caractérisent, s'étendent aux cellules voisines via ces communications. Pour éviter cela, il existe un mécanisme de contrôle d'urgence : la fermeture des canaux en présence d'une concentration élevée de calcium, anomalie constante en cas de lésion cellulaire, ou d'une modification brutale du potentiel de membrane. Le pire, c'est peut-être aussi la diffusion dans une culture de cellules sénescentes de petites molécules à action antiproliférative, accumulées au départ dans certaines seulement des cellules.

Un tel contrôle de la prolifération cellulaire par les cellules adjacentes, via des substances diffusant par les canaux jonctionnels, est d'ailleurs probable en dehors même des phénomènes de sénescence. Lorsque sont co-cultivées des cellules normales et transformées (c'est-à-dire ayant un phénotype "malin " et tumorigène) échangeant peu de canaux jonctionnels, les cellules transformées prolifèrent activement ; lorsque de I'AMP cyclique (qui catalyse la phosphorylation de protéines intervenant dans la constitution des canaux) est ajouté aux cultures, l'établissement des jonctions intercellulaires est corrélé à l'inhibition de la prolifération des cel- 
lules malignes. Au contraire, une combinaison de cellules normales et transformées établissant spontanément de nombreux canaux jonctionnels ne prolifère pas, à moins que les canaux ne soient bloqués par l'addition d'acide rétinoïque [5].

Il est possible que les cellules malignes, pour échapper à l'influence inhibitrice sur leur prolifération de cellules normales adjacentes, aient souvent à bloquer les communications intercellulaires. Dans les cellules transformées par le virus de Rous, le déficit en communications intercellulaires est ainsi étroitement parallèle à l'activité de la "tyrosine-kinase " p60 $60^{\mathrm{v} \text {-src }}[1,6]$. L'oncogène moyen $T$ de polyome dont l'effet transformant pourrait être relié à son aptitude à lier et à activer la p60 c-src $\left(m / s \quad n^{\circ} 8\right.$, vol. 2, p. 465) inhibe aussi l'établissement des communications intercellulaires.

Ainsi, les canaux de jonction intercellulaires, facteurs de communication directe entre les cellules et de circulation des ions et des petites molécules, sont-ils impliqués dans l'essentiel des phénomènes cellulaires : prolifération, transformation, morphogenèse et différenciation, sécrétion... et peut-être sénescence.

Axel Kahn

\section{RÉFÉRENCES}

1. Loewenstein WR. The cell-to-cell channel of gap junction. Cell 1987 ; 48 : 735-6. 2. Bargiello TA, Saez L, Baylies MK, Gasic G, Young MW, Spray DC. The drosophila clock gene per affects intercellular junctional communication. Nalure 1987 ; 328 ; 686-91. 3. Spray DC, Fujita M, Saez JC, et al. Proteoglycans and glycosaminoglycans induce gap junction synthesis and function on primary liver cultures. J Cell Biol 1987 ; 105 : 541-51.

4. Meda P, Buzzone R, Chanson M, Bosco $\mathrm{D}$, Orci L. Gap junctional coupling modulates secretion of exocrine pancreas. Proc Nall Acad Sci USA 1987 ; 84 : 4901-4.

5. Mehta P, Bertram JS, Loewenstein WR. Growth inhibition of transformed cells correlates with their junctional communication with normal cells. Cell 1986 ; 44 : 187-96.

6. Azarnia R, Loewenstein WR. Polyoma virus middle $\mathrm{T}$ antigen downregulates junctional cell-to-cell communication. Mol Cell Biol 1987 ; 7 : 946-50.

$\mathrm{m} / \mathrm{s} n^{\circ} 9$ vol. 3 , novembre 87

\section{口曰 BRÈVES}

- L'essai randomisé et prospectif de Birmingham effectué chez plus de 100 enfants ayant un reflux vésico-urétéral important compare les effets de la correction chirurgicale à ceux d'un traitement non opératoire, après deux et cinq ans d'observation. Il n'y a aucune différence entre les 2 groupes, concernant la fréquence des infections, la fonction et la croissance des reins, et la formation ou la progression des lésions rénales (malgré la persistance du reflux chez plus de $50 \%$ des enfants traités médicalement). Peut-on identifier encore plus tôt les enfants à risque, avant l'apparition des lésions rénales?

[Birmingham reflux study group. Br Med J 1987 ; 295 : 237-41]

L'un des gènes de susceptibilité au diabète chez la souris code pour une molécule de classe II du complexe majeur d'histocompatibilité. La souris NOD (non-obese diabetic) développe spontanément un diabète sucré avec atteinte auto-immune des ilôts de Langerhans. Une étude génétique a permis de démontrer que la susceptibilité au diabète était sous le contrôle de deux gènes dont l'un est sur le chromosome 17, génétiquement lié au complexe majeur d'histocompatibilité (CMH). Les souris NOD ont par ailleurs des anomalies de l'expression des molécules de classe II du CMH : elles ne synthétisent pas la chaîne $\alpha$ des molécules I-E. La correction de cette anomalie par introduction d'un transgène codant pour I-E supprime la prédisposition au diabète des souris NOD transgéniques. Il existe donc une relation directe entre les molécules du $\mathrm{CMH}$ et le diabète auto-immun. Cette conclusion pourrait être également valable chez l'homme dont la prédisposition au diabète juvénile insulino-dépendant est génétiquement liée au locus HLA-DR. [Nishimoto H, et al. Nature 1987 ; $328: 432-4]$
Quelle différence y-a-t-il entre l'interaction avec le récepteur, des stéroïdes et des antistéroïdes? Les deux types de molécule ont habituellement la même affinité pour le récepteur et, in vitro, le récepteur, libre ou bien complexé soit au stéroïde, soit à l'antagoniste, semble se fixer de façon similaire aux séquences spécifiques d'ADN. In vivo, cependant, tous les résultats expérimentaux montrent que seul le complexe récepteur-hormone se fixe efficacement à ses cibles d'ADN. André Groyer et al., dans le laboratoire du Pr. E. Baulieu, viennent de démontrer qu'in vivo, le récepteur des glucocorticoïdes (RG) était complexé à la protéine hsp90 (protéine du choc thermique, d'un poids moléculaire de 90000 ) et n'était pas, sous cette forme, disponible pour se fixer aux éléments régulateurs des gènes modulés par les glucocorticoïdes. Les agonistes des glucocorticoïdes libèrent le RG de ce complexe, alors qu'au contraire les antagonistes (par exemple, le RU 489) le stabilisent.

[Groyer A, et al. Nature 1987 $328 ; 624-6$ ]

La signification de l'activation des oncogènes ras n'est pas toujours évidente! Ainsi, une double mutation du codon 61 des oncogènes $\mathrm{N}$-ras et $\mathrm{Ki}$-ras vient-elle d'être décrite dans les cellules leucémiques de deux malades atteints de leucémie aiguë myéloblastique. Cependant, alors que ces anomalies existaient in vivo dans un cas, elles étaient apparues au cours de l'établissement d'une lignée cellulaire en culture dans l'autre cas; dans cette dernière observation, il n'existait pas de différence morphologique ou de capacité proliférative entre les cellules possédant ou non cette double mutation. [Janssen JWG, et al. Nucleic Acids Res 1987; 15 : 5669-80] 